\title{
A Penalized-Equation-Based Generalized Newton Method for Solving Absolute-Value Linear Complementarity Problems
}

\author{
Yuan Li, Hai-Shan Han, and Dan-Dan Yang \\ College of Mathematics, Inner Mongolia University for the Nationalities, The Inner Mongolia Autonomous Region, \\ Tongliao 028000, China \\ Correspondence should be addressed to Yuan Li; imunliyuan@163.com
}

Received 7 June 2014; Accepted 26 August 2014; Published 9 September 2014

Academic Editor: Liwei Zhang

Copyright (C) 2014 Yuan Li et al. This is an open access article distributed under the Creative Commons Attribution License, which permits unrestricted use, distribution, and reproduction in any medium, provided the original work is properly cited.

We consider a class of absolute-value linear complementarity problems. We propose a new approximation reformulation of absolute value linear complementarity problems by using a nonlinear penalized equation. Based on this approximation reformulation, a penalized-equation-based generalized Newton method is proposed for solving the absolute value linear complementary problem. We show that the proposed method is globally and superlinearly convergent when the matrix of complementarity problems is positive definite and its singular values exceed 1 . Numerical results show that our proposed method is very effective and efficient.

\section{Introduction}

Let $F: \Re^{n} \rightarrow \Re^{n}$ be a given function. The complementarity problems, $\mathrm{CP}(F)$ for short, is to find a solution of the system

$$
x \leq 0, \quad F(x) \leq 0, \quad F(x)^{T} x=0 .
$$

The $\mathrm{CP}(F)$ is called the linear complementarity problems (for short LCP) if $F$ is an affine mapping of the form

$$
F(x)=A x-b,
$$

where $A \in \Re^{n \times n}$ and $b \in \mathfrak{R}^{n}$. Otherwise, the $\mathrm{CP}(F)$ is called the nonlinear complementarity problems $(\mathrm{NCP}(F))$.

The systematic study of the finite-dimensional $\mathrm{CP}(F)$ began in the mid-1960s; in a span of five decades, the subject has developed into a very fruitful discipline in the field of mathematical programming. The developments include a rich mathematical theory, a host of effective solution algorithms, a multitude of interesting connections to numerous disciplines, and a wide range of important applications in engineering and economics (see, e.g., [1-4] and the references therein).

Generalized Newton method (semismooth Newton method) is one of efficacious algorithms for solving $\mathrm{CP}(F)$.
The main idea of semismooth Newton method is based on an equivalent reformulation of the complementarity problems consisting of a nonsmooth equation and then solving the nonsmooth equation by Newton type method (see, e.g., $[5,6])$. Most reformulations of the $\mathrm{CP}(F)$ are based on the Fischer-Burmeister function [7] (see, e.g., [8-10] and the references therein). Chen et al. [11] introduced a penalized Fischer-Burmeister function and proposed a new semismooth Newton method based on this new NCP function. Kanzow and Kleinmichel [12] proposed a new, one-parametric class of NCP functions based on FischerBurmeister function and gave a semismooth Newton method via these NCP functions. Kanzow [13] researched an inexact semismooth Newton method based on Fischer-Burmeister function and penalized Fischer-Burmeister function. Ito and Kunisch [14] studied a semismooth Newton method based on the max-type NCP function.

All generalized Newton methods mentioned above involve the continuously differentiable assumption on $F$ in $\mathrm{CP}(F)$. The existed generalized Newton methods proposed are based on the equivalent reformulation via NCP functions. To the best of our knowledge, until now, there exist very few literature resources to study the complementary problems when the involved function $F$ is not differentiable. However, 
as in many practical problems, $F$ is not differentiable; for instance, $F(x)=A x-|x|-b$; see Noor et al. [15]. This is our focus in this paper.

In this paper, we consider the following absolute-value linear complementarity problems (for short $\operatorname{AVLCP}(A, b)$ ): find $x \in \Re^{n}$ such that

$$
x \leq 0, \quad A x-|x|-b \leq 0, \quad(A x-|x|-b)^{T} x=0,
$$

where $A \in \mathfrak{R}^{n \times n}$ and $b \in \mathfrak{R}^{n}$.

The $\operatorname{AVLCP}(A, b)$ is a special case when $F$ is a piecewiselinear function $F=A x-|x|-b$ in $\mathrm{CP}(F)$ and can be viewed as an extension of the LCP. This complementarity problem was first introduced and studied by Noor et al. [15] in 2012. Noor et al. proposed a generalized AOR method via establishing the equivalence between $\operatorname{AVLCP}(A, b)$ and the fixed point problem using the projection operator, but the convergence rate is linear. Moreover, the study on $\operatorname{AVLCP}(A, b)$ is in its infancy and, to the authors knowledge, there has been no work except for the above-mentioned results of Noor et al. [15]. These observations motivated us to further improve the theory and numerical system for solving $\operatorname{AVLCP}(A, b)$.

We use the penalty technique to show that the $\operatorname{AVLCP}(A, b)$ in $\mathfrak{R}^{n}$ are approximately equivalent to a nonlinear penalized equation, which was first introduced for solving $\operatorname{AVLCP}(A, b)$. It is worth mentioning that the penalty technique has been widely used in solving nonlinear programming, but it seems that there is a limited study for complementarity problems (see [16-18]). We show that the solution to this penalized equation converges to that of the $\operatorname{AVLCP}(A, b)$ at an exponential rate when the penalized parameter tends to infinity. We again use the generalized Jacobian based on subgradient to analyze a generalized Newton method for solving the nonlinear penalized equation under some mild assumptions. The algorithm will be shown to be superlinearly convergent and can start from an arbitrary point. Preliminary numerical experiments are also given to show the effectiveness and efficiency of the proposed method.

The rest of this paper is organized as follows. In Section 2, we present some notations and the well-known results. In Section 3, we provide a penalized equation for approximating the $\operatorname{AVLCP}(A, b)$ and its properties. In Section 4, a generalized Newton method is introduced for solving the penalized equation. In Section 5, we introduce the numerical results of our methods.

\section{Preliminaries}

For convenience, we will now briefly explain some of the terminologies that will be used in the next section. $\mathfrak{R}^{n}$ denotes the $n$-dimensional Euclidean space. All vectors in $\Re^{n}$ are column vectors. Let $A=\left(a_{i j}\right) \in \mathfrak{R}^{n \times n}$ be an $n \times n$ real matrix. The scalar product of two vectors $x$ and $y$ is denoted by $x^{T} y$. For $p>1$, the $p$-norm of $x=\left(x_{1}, x_{2}, \ldots, x_{n}\right)^{T} \in$ $\mathfrak{R}^{n}$ is defined as $\|x\|_{p}=\left(\sum_{i=1}^{n}\left|x_{i}\right|^{p}\right)^{1 / p}$. When $p=2$, the $p$-norm becomes the 2-norm $\|x\|=\left(x^{T} x\right)^{1 / 2}$. For any $x=\left(x_{1}, x_{2}, \ldots, x_{n}\right) \in \mathfrak{R}^{n}$ and $\alpha \in \Re, x^{\alpha}=\left(x_{1}^{\alpha}, x_{2}^{\alpha}, \ldots, x_{n}^{\alpha}\right)$. $|x|$ stands for the vector in $\Re^{n}$ of absolute values of components of $x \cdot \operatorname{sign}(x)$ will denote the vector with components equal to 1,0 , or -1 depending on whether the corresponding component of $x$ is positive, zero, or negative. $\operatorname{diag}(\operatorname{sign}(x))$ will denote a diagonal matrix corresponding to $\operatorname{sign}(x)$. The plus function $[x]_{+}$, which replaces the negative components of $x$ by zeros, is a projection operator that projects $x$ onto the nonnegative orthant; namely, $[x]_{+}=\max \{x, 0\}$. For a solvable matrix equation $B y=d$, we will use the MATLAB backslash $B \backslash d$ to denote a solution $y$. The generalized Jacobians $\partial|x|$ of $|x|$ and $\partial[x]_{+}^{1 / l}$ of $[x]_{+}^{1 / l}$ based on a subgradient [20] of their components are given by the diagonal matrices $D(x)$ and $D^{\prime}(x)$, respectively, where $D(x)=\operatorname{diag}(\operatorname{sign}(x))$ and $D^{\prime}(x)$ is the the diagonal matrix whose diagonal entries are equal to $(1 / l) x_{i}^{(1 / l)-1}, 0$, or a real number $\sigma \in[0,1]$ depending on whether the corresponding component of $x$ is positive, negative, or zero.

Definition 1 (see [21]). Let $A=\in \mathfrak{R}^{n \times n}$ be a matrix; the matrix $A$ is called

(1) positive definite if there exists a constant $\gamma>0$ such that $x^{T} A x \geq \gamma\|x\|^{2}$ for any $x \in \mathfrak{R}^{n}$,

(2) bounded if there exists a constant $\beta>0$ such that $\|A x\| \leq \beta\|x\|$ for any $x \in \mathfrak{R}^{n}$.

Lemma 2 (Hölder's inequality). Let $x, y \in \mathfrak{R}^{n}$. Then

$$
\left|x^{T} y\right| \leq\|x\|_{p}\|y\|_{q}=\left(\sum_{i=1}^{n}\left|x_{i}\right|^{p}\right)^{1 / p}\left(\sum_{i=1}^{n}\left|y_{i}\right|^{q}\right)^{1 / q},
$$

where $p>1$ and $q>1$ are real numbers such that $(1 / p)+$ $(1 / q)=1$.

Lemma 3 (see [15]). Let $K$ be a closed and convex set in $\mathfrak{R}^{n}$. $A$ vector $x$ solves the $\operatorname{AVLCP}(A, b)$ if and only if $x$ solves the following absolute-value variational inequalities:

$$
\langle A x-|x|-b, y-x\rangle \geq 0, \quad \forall y \in K \text {. }
$$

Lemma 4. Let $A \in \mathfrak{R}^{m \times n}, x \in \mathfrak{R}^{n}$. Then

$$
\|A x\| \leq\|A\|_{F}\|x\|
$$

where $\|A\|_{F}$ denotes the Frobenius norm $\|A\|_{F}^{2}=\operatorname{tr}\left(A^{T} A\right)=$ $\sum_{i=1}^{m} \sum_{j=1}^{n}\left|a_{i j}\right|^{2}$.

Lemma 5 (see [22]). The singular values of the matrix $A \in$ $\mathfrak{R}^{n \times n}$ exceed 1 if and only if the minimum eigenvalue of $A^{T} A$ exceeds 1 .

Lemma 6 ((Banach perturbation lemma) [21]). Let $A, C \in$ $\mathfrak{R}^{n \times n}$ and assume that $A$ is invertible with $\left\|A^{-1}\right\| \leq \alpha$. If $\| A-$ $C \| \leq \beta$ and $\alpha \beta<1$, then $C$ is also invertible and

$$
\left\|C^{-1}\right\| \leq \frac{\alpha}{1-\alpha \beta} .
$$


Lemma 7 (see [23]). Let $x, y \in \mathfrak{R}^{n}$. Then

$$
\||x|-|y|\| \leq 2\|x-y\| \text {. }
$$

Lemma 8. Let $x, y \in \mathfrak{R}^{n}$. Then

$$
\left\|[x]_{+}-[y]_{+}\right\| \leq\|x-y\| .
$$

\section{A Penalized-Equation Approximation Reformulation of $\operatorname{AVLCP}(A, b)$}

In this section, we construct a nonlinear penalized equation corresponding to absolute-value linear complementarity problem (3).

Find $x_{\lambda} \in \mathfrak{R}^{n}$ such that

$$
A x_{\lambda}-\left|x_{\lambda}\right|+\lambda\left[x_{\lambda}\right]_{+}^{1 / \ell}=b,
$$

where $\lambda>1$ is the penalized parameter and $\ell \geq 1$.

We will prove that the solution to the penalized equation (10) converges to that of the $\operatorname{AVLCP}(A, b)$. Thus, we make the following assumptions on the system matrix $A$ :

(A1) $A$ is positive definite and $\gamma>1$ in Definition 1;

(A2) the entries of $A$ satisfy $a_{i i}>0, a_{i j} \leq 0$ for all $i, j=$ $1,2, \ldots, n$ with $i \neq j$.

Under assumption (A1), the solution of $\operatorname{AVLCP}(A, b)$ is unique [15]. Our main results in this section are as follows. First, we start our discussion with the following lemma.

Lemma 9. Let $x_{\lambda}$ be the solution to nonlinear penalized equation (10). Then there exists a positive constant $C_{0}$, independent of $n, x_{\lambda}$, and $\lambda$ such that

$$
\left\|\left[x_{\lambda}\right]_{+}\right\| \leq \frac{C_{0}}{\lambda^{\ell / 2}}
$$

where $\lambda$ and $\ell$ are parameters used in (10).

Proof. Left-multiplying both sides of (10) by $\left[x_{\lambda}\right]_{+}^{T}$ gives

$$
\left[x_{\lambda}\right]_{+}^{T} A x_{\lambda}-\left[x_{\lambda}\right]_{+}^{T}\left|x_{\lambda}\right|+\lambda\left[x_{\lambda}\right]_{+}^{T}\left[x_{\lambda}\right]_{+}^{1 / \ell}=\left[x_{\lambda}\right]_{+}^{T} b .
$$

Without loss of generality, we assume that $\left[x_{\lambda}\right]_{+}=\left(u_{1}^{T}, 0\right)^{T}$, where $u_{1} \in \mathfrak{R}^{m}, 0 \leq m \leq n, u_{1} \geq 0$. Other cases can be transformed into this by reordering the system.

When $m=0$, then $\left[x_{\lambda}\right]_{+}=0$; thus (11) is trivially satisfied. We only consider the case when $m \geq 1$. In this case $x_{\lambda}$ can be decomposed into $x_{\lambda}=\left(u_{1}^{T}, u_{2}^{T}\right)^{T}$, where $u_{2} \in \mathfrak{R}^{n-m}, u_{2} \leq 0$, We now decompose $A$ into

$$
A=\left(\begin{array}{ll}
A_{11} & A_{12} \\
A_{21} & A_{22}
\end{array}\right)
$$

where $A_{11} \in \mathfrak{R}^{m \times m}, A_{12} \in \mathfrak{R}^{m \times(n-m)}, A_{21} \in \mathfrak{R}^{(n-m) \times m}$, and $A_{22} \in \Re^{(n-m) \times(n-m)}$; then (12) becomes

$$
u_{1}^{T} A_{11} u_{1}+u_{1}^{T} A_{12} u_{2}-\left[x_{\lambda}\right]_{+}^{T}\left|x_{\lambda}\right|+\lambda\left\|\left[x_{\lambda}\right]_{+}\right\|_{p}^{p}=\left[x_{\lambda}\right]_{+}^{T} b,
$$

where $p=1+(1 / \ell)$.
From assumption (A1) and $u_{1} \geq 0$, we have $u_{1}^{T} A_{11} u_{1} \geq 0$, and

$$
u_{1}^{T} A_{11} u_{1}=\left[x_{\lambda}\right]_{+}^{T} A\left[x_{\lambda}\right]_{+} \geq \gamma\left\|\left[x_{\lambda}\right]_{+}\right\|^{2} \geq\left\|\left[x_{\lambda}\right]_{+}\right\|^{2}
$$

holds, where the last inequality follows from $\gamma>1$. From assumption (A2) and $u_{2} \leq 0$, we also have $u_{1}^{T} A_{12} u_{2} \geq 0$. Note that $\left[x_{\lambda}\right]_{+}^{T}\left|x_{\lambda}\right|=\left\|\left[x_{\lambda}\right]_{+}\right\|^{2}$; one has that

$$
u_{1}^{T} A_{11} u_{1}-\left[x_{\lambda}\right]_{+}^{T}\left|x_{\lambda}\right| \geq 0 .
$$

It follows from (14), (16), and $u_{1}^{T} A_{12} u_{2} \geq 0$ that we have the following inequality:

$$
\lambda\left\|\left[x_{\lambda}\right]_{+}\right\|_{p}^{p} \leq\left[x_{\lambda}\right]_{+}^{T} b .
$$

By using Lemma 2 in (17), we get

$$
\lambda\left\|\left[x_{\lambda}\right]_{+}\right\|_{p}^{p} \leq\left[x_{\lambda}\right]_{+}^{T} b \leq\left\|\left[x_{\lambda}\right]_{+}\right\|_{p}\|b\|_{q},
$$

where $q=\ell+1$ satisfying $(1 / p)+(1 / q)=1$. Since $p-1=1 / \ell$, we thus have the following inequality:

$$
\left\|\left[x_{\lambda}\right]_{+}\right\|_{p} \leq \frac{1}{\lambda^{\ell}}\|b\|_{q}^{\ell} \text {. }
$$

Since all norms on $\mathfrak{R}^{n}$ are equivalent for a fixed positive integer $n$, then it follows that there exists a positive constant $a_{0}$ such that

$$
\left\|\left[x_{\lambda}\right]_{+}\right\| \leq a_{0}\left\|\left[x_{\lambda}\right]_{+}\right\|_{p}
$$

and thus the left hand in (16) can be written as

$$
\begin{aligned}
& u_{1}^{T} A_{11} u_{1}+u_{1}^{T} A_{12} u_{2}-\left[x_{\lambda}\right]_{+}^{T}\left|x_{\lambda}\right|+\lambda\left\|\left[x_{\lambda}\right]_{+}\right\|_{p}^{p} \\
& \geq u_{1}^{T} A_{11} u_{1}+u_{1}^{T} A_{12} u_{2}-\left[x_{\lambda}\right]_{+}^{T}\left|x_{\lambda}\right|+\frac{\lambda}{a_{0}^{p}}\left\|\left[x_{\lambda}\right]_{+}\right\|^{p} .
\end{aligned}
$$

Combining with (14), we obtain

$$
u_{1}^{T} A_{11} u_{1}+u_{1}^{T} A_{12} u_{2}-\left[x_{\lambda}\right]_{+}^{T}\left|x_{\lambda}\right|+\frac{\lambda}{a_{0}^{p}}\left\|\left[x_{\lambda}\right]_{+}\right\|^{p} \leq\left[x_{\lambda}\right]_{+}^{T} b,
$$

together with (16); dropping the first three terms in (22) and using Lemma 2, we have

$$
\frac{\lambda}{a_{0}^{p}}\left\|\left[x_{\lambda}\right]_{+}\right\|^{p} \leq\left\|\left[x_{\lambda}\right]_{+}\right\|_{p}\|b\|_{q} .
$$

Since $p=1+(1 / \ell) \in(1,2]$, we thus have from the above inequality

$$
\left\|\left[x_{\lambda}\right]_{+}\right\| \leq \frac{1}{\lambda^{(\ell+1) / p}}\left(a_{0}^{p}\|b\|_{q}^{\ell+1}\right)^{1 / p} \leq \frac{1}{\lambda^{\ell / 2}}\left(a_{0}^{p}\|b\|_{q}^{\ell+1}\right)^{1 / p}=\frac{C_{0}}{\lambda^{\ell / 2}},
$$

where $C_{0}=\left(a_{0}^{p}\|b\|_{q}^{\ell+1}\right)^{1 / p}$. Thus, the proof of this lemma is completed. 
Using Lemma 9, we can establish the relationship between solutions of penalized equation (10) and solutions of the $\operatorname{AVLCP}(A, b)$.

Theorem 10. Let $x^{*}$ and $x_{\lambda}$ be the solution to $\operatorname{AVLCP}(A, b)$ and nonlinear penalized equation (10), respectively. Then there exists a positive constant $C$, independent of $n, x^{*}, x_{\lambda}$, and $\lambda$ such that

$$
\left\|x^{*}-x_{\lambda}\right\| \leq \frac{C}{\lambda^{\ell / 2}}
$$

where $\lambda$ and $\ell$ are parameters used in (10).

Proof. Let $\left[x_{\lambda}\right]_{-}=-\min \left\{x_{\lambda}, 0\right\}$; then

$$
x_{\lambda}=\left[x_{\lambda}\right]_{+}-\left[x_{\lambda}\right]_{-} \text {, }
$$

and the vector $x^{*}-x_{\lambda}$ can be decomposed as

$$
x^{*}-x_{\lambda}=x^{*}+\left[x_{\lambda}\right]_{-}-\left[x_{\lambda}\right]_{+}=r_{\lambda}-\left[x_{\lambda}\right]_{+},
$$

where $r_{\lambda}=x^{*}+\left[x_{\lambda}\right]_{-}$.

Let $\omega=x^{*}-r_{\lambda}$; one has that

$$
\omega=x^{*}-r_{\lambda}=-\left[x_{\lambda}\right]_{-} \leq 0,
$$

and therefore $\omega \in K=\left\{y \in \mathfrak{R}^{n} \mid y \leq 0\right\}$.

Replacing $y$ in (5) by $\omega$ gives

$$
-r_{\lambda}^{T}\left(A x^{*}-\left|x^{*}\right|\right) \geq-r_{\lambda}^{T} b
$$

left-multiplying (10) by $r_{\lambda}^{T}$ we have

$$
r_{\lambda}^{T} A x_{\lambda}-r_{\lambda}^{T}\left|x_{\lambda}\right|+\lambda r_{\lambda}^{T}\left[x_{\lambda}\right]_{+}^{1 / \ell}=r_{\lambda}^{T} b ;
$$

adding up to (29) and (30), we get

$$
r_{\lambda}^{T} A\left(x_{\lambda}-x^{*}\right)+r_{\lambda}^{T}\left(\left|x^{*}\right|-\left|x_{\lambda}\right|\right)+\lambda r_{\lambda}^{T}\left[x_{\lambda}\right]_{+}^{1 / \ell} \geq 0 ;
$$

further we get from (31) that

$$
r_{\lambda}^{T} A\left(x_{\lambda}-x^{*}\right)+r_{\lambda}^{T}\left(\left|x^{*}\right|-\left|x_{\lambda}\right|\right) \geq 0,
$$

where

$$
r_{\lambda}^{T}\left[x_{\lambda}\right]_{+}^{1 / \ell}=\left(x^{*}+\left[x_{\lambda}\right]_{-}\right)^{T}\left[x_{\lambda}\right]_{+}^{1 / \ell}=\left(x^{*}\right)^{T}\left[x_{\lambda}\right]_{+}^{1 / \ell} \leq 0 .
$$

One further has that

$$
\begin{aligned}
& r_{\lambda}^{T} A\left(x_{\lambda}-x^{*}\right)+r_{\lambda}^{T}\left(\left|x^{*}\right|-\left|x_{\lambda}\right|\right) \\
& \quad=r_{\lambda}^{T} A\left(x_{\lambda}-x^{*}\right)+r_{\lambda}^{T}\left(-x^{*}-\left|x_{\lambda}\right|+x_{\lambda}-x_{\lambda}\right) \\
& \quad=r_{\lambda}^{T} A\left(x_{\lambda}-x^{*}\right)+r_{\lambda}^{T}\left(x_{\lambda}-x^{*}\right)-r_{\lambda}^{T}\left(x_{\lambda}+\left|x_{\lambda}\right|\right) \\
& \quad=r_{\lambda}^{T} A\left(x_{\lambda}-x^{*}\right)+r_{\lambda}^{T}\left(x_{\lambda}-x^{*}\right)-2 r_{\lambda}^{T}\left[x_{\lambda}\right]_{+} \\
& \quad=r_{\lambda}^{T} A\left(\left[x_{\lambda}\right]_{+}-r_{\lambda}\right)+r_{\lambda}^{T}\left(\left[x_{\lambda}\right]_{+}-r_{\lambda}\right)-2 r_{\lambda}^{T}\left[x_{\lambda}\right]_{+} \\
& \quad=r_{\lambda}^{T} A\left[x_{\lambda}\right]_{+}-r_{\lambda}^{T} A r_{\lambda}-r_{\lambda}^{T} r_{\lambda}-r_{\lambda}^{T}\left[x_{\lambda}\right]_{+} ;
\end{aligned}
$$

thus

$$
r_{\lambda}^{T} A\left[x_{\lambda}\right]_{+}-r_{\lambda}^{T}\left[x_{\lambda}\right]_{+} \geq r_{\lambda}^{T} A r_{\lambda}+r_{\lambda}^{T} r_{\lambda} \geq(\gamma+1)\left\|r_{\lambda}\right\|^{2} ;
$$

the last inequality is true by the positive definiteness of $A$.

Hence

$$
r_{\lambda}^{T} A\left[x_{\lambda}\right]_{+}-r_{\lambda}^{T}\left[x_{\lambda}\right]_{+} \geq 0 .
$$

On the other hand, using the Cauchy-Schwarz inequality, Lemma 4, and (11) to (36), we get

$$
\begin{array}{rl}
r_{\lambda}^{T} & A\left[x_{\lambda}\right]_{+}-r_{\lambda}^{T}\left[x_{\lambda}\right]_{+} \\
& \leq\left|r_{\lambda}^{T} A\left[x_{\lambda}\right]_{+}\right|+\left|r_{\lambda}^{T}\left[x_{\lambda}\right]_{+}\right| \\
& \leq\left\|r_{\lambda}\right\| \cdot\left\|A\left[x_{\lambda}\right]_{+}\right\|+\left\|r_{\lambda}\right\| \cdot\left\|\left[x_{\lambda}\right]_{+}\right\| \\
& \leq\left\|r_{\lambda}\right\| \cdot\|A\|_{F} \cdot\left\|\left[x_{\lambda}\right]_{+}\right\|+\left\|r_{\lambda}\right\| \cdot\left\|\left[x_{\lambda}\right]_{+}\right\| \\
& =\left\|r_{\lambda}\right\| \cdot\left\|\left[x_{\lambda}\right]_{+}\right\| \cdot\left(\|A\|_{F}+1\right) \\
& \leq\left\|r_{\lambda}\right\| \cdot \frac{C_{0}}{\lambda^{\ell / 2}} \cdot\left(\|A\|_{F}+1\right) ;
\end{array}
$$

this implies that

$$
\left\|r_{\lambda}\right\| \leq \frac{C_{0}\left(\|A\|_{F}+1\right)}{(\gamma+1) \lambda^{\ell / 2}}=\frac{C^{\prime}}{\lambda^{\ell / 2}},
$$

where $C^{\prime}=C_{0}\left(\|A\|_{F}+1\right) /(\gamma+1)$. From (11), (27), (38), and the triangle inequality, we obtain

$$
\left\|x^{*}-x_{\lambda}\right\| \leq\left\|r_{\lambda}\right\|+\left\|\left[x_{\lambda}\right]_{+}\right\| \leq \frac{C}{\lambda^{\ell / 2}} .
$$

We complete this theorem.

\section{A Penalized-Equation-Based Generalized Newton Method and Its Convergence}

In this section, we present a generalized Newton method for solving nonlinear penalized equation (10). We begin by defining the vector function specified by the nonlinear penalized equation (10) as follows:

$$
g\left(x_{\lambda}\right)=A x_{\lambda}-\left|x_{\lambda}\right|+\lambda\left[x_{\lambda}\right]_{+}^{1 / \ell}-b, \quad \lambda>1 .
$$

Let $x_{\lambda}=\left(u_{1}, u_{2}, \ldots, u_{n}\right)^{T}$; a generalized Jacobian $\partial g\left(x_{\lambda}\right)$ of $g\left(x_{\lambda}\right)$ is given by

$$
\partial g\left(x_{\lambda}\right)=A-D\left(x_{\lambda}\right)+\lambda D^{\prime}\left(x_{\lambda}\right)
$$

where $D\left(x_{\lambda}\right)=\partial\left|x_{\lambda}\right|=\operatorname{diag}\left(\operatorname{sign}\left(x_{\lambda}\right)\right)$ and $D^{\prime}\left(x_{\lambda}\right)=$ $\partial\left[x_{\lambda}\right]_{+}^{1 / \ell}$ is a diagonal matrix whose diagonal entries are equal to $(1 / \ell) u_{i}^{(1 / \ell)-1}, 0$, or a real number $\sigma \in[0,1]$ depending on whether the corresponding component of $x_{\lambda}$ is positive, negative, or zero. The generalized Newton method for finding a solution of the equation $g\left(x_{\lambda}\right)=0$ consists of the following iteration:

$$
g\left(x_{\lambda}^{i}\right)+\partial g\left(x_{\lambda}^{i}\right)\left(x_{\lambda}^{i+1}-x_{\lambda}^{i}\right)=0 .
$$


Replacing $g\left(x_{\lambda}^{i}\right)$ by its definition (40) and setting $\partial g\left(x_{\lambda}^{i}\right)$ by (41) give

$$
\left[A-D\left(x_{\lambda}^{i}\right)+\lambda D^{\prime}\left(x_{\lambda}^{i}\right)\right] x_{\lambda}^{i+1}=b+\lambda\left(\frac{1}{\ell}-1\right)\left[x_{\lambda}^{i}\right]_{+}^{1 / \ell} .
$$

Thus, solving for $x_{\lambda}^{i+1}$ gives

$$
x_{\lambda}^{i+1}=\left[A-D\left(x_{\lambda}^{i}\right)+\lambda D^{\prime}\left(x_{\lambda}^{i}\right)\right] \backslash\left(b+\lambda\left(\left(\frac{1}{\ell}\right)-1\right)\left[x_{\lambda}^{i}\right]_{+}^{1 / \ell}\right),
$$

which is our final generalized Newton iteration for solving the nonlinear penalized equation (10). In the following, we can establish the penalized-equation-based generalized Newton method for solving $\operatorname{AVLCP}(A, b)$.

Algorithm 11 (penalized-equation-based generalized Newton algorithm). We have the following.

Step 1. Given constants $\varepsilon>0, \lambda_{0}>1, \mu>1, \ell \geq 1$, and $\sigma \in[0,1]$ and a starting point $x_{\lambda_{0}}^{0} \in \mathfrak{R}^{n}$, set $k:=0$.

Step 2. Calculate $x_{\lambda_{k}}^{i+1}$ from the generalized Newton equation

$$
\left[A-D\left(x_{\lambda_{k}}^{i}\right)+\lambda_{k} D^{\prime}\left(x_{\lambda_{k}}^{i}\right)\right] x_{\lambda_{k}}^{i+1}=b+\lambda_{k}\left(\frac{1}{\ell}-1\right)\left[x_{\lambda_{k}}^{i}\right]_{+}^{1 / \ell},
$$

starting from $x_{\lambda_{k}}^{0}$ associated with $\lambda_{k}$.

Step 3. If $D\left(x_{\lambda_{k}}^{i+1}\right)=D\left(x_{\lambda_{k}}^{i}\right), D^{\prime}\left(x_{\lambda_{k}}^{i+1}\right)=D^{\prime}\left(x_{\lambda_{k}}^{i}\right)$ and $\| x_{\lambda_{k}}^{i+1}-$ $x_{\lambda_{k}}^{i} \| \leq \varepsilon$, stop; otherwise, set $i:=i+1$ and go to Step 2. Denote the accumulation point of $\left\{x_{\lambda_{k}}^{i}\right\}$ by $\bar{x}_{k}$.

Step 4. If $\left\|\left[\bar{x}_{k}\right]_{+}\right\| \leq \varepsilon$, then stop; otherwise, let $\lambda_{k+1}=\mu \lambda_{k}$, choose new starting point $x_{\lambda_{k+1}}^{0}=\bar{x}_{k}$, set $k:=k+1$, and go to Step 2.

4.1. Existence of Accumulation Point at Each Generalized Newton Iteration. We will show that the sequence $\left\{x_{\lambda_{k}}^{i}\right\}_{i=1}^{+\infty}$ generated by generalized Newton iteration (45) converges to an accumulation point $\bar{x}_{k}$ associated with $\lambda_{k}$. We firstly give the following sufficient conditions that the generalized Newton iteration (45) is well defined.

Lemma 12. If the singular values of $A \in \mathfrak{R}^{n \times n}$ exceed 1 . Then $\left(A-D\left(x_{\lambda_{k}}^{i}\right)+\lambda_{k} D^{\prime}\left(x_{\lambda_{k}}^{i}\right)\right)^{-1}$ exists for any diagonal matrices $D\left(x_{\lambda_{k}}^{i}\right)$ and $D^{\prime}\left(x_{\lambda_{k}}^{i}\right)$, where $\lambda_{k}>1, D\left(x_{\lambda_{k}}^{i}\right)$ is the diagonal matrix with diagonal elements equal to \pm 1 or 0 and $D^{\prime}\left(x_{\lambda_{k}}^{i}\right)$ is the diagonal matrix with diagonal elements equal to $(1 / \ell) u_{i}^{(1 / \ell)-1}, 0$, or a real number $\sigma \in[0,1]$.

Proof. If $\left(A-D\left(x_{\lambda_{k}}^{i}\right)+\lambda_{k} D^{\prime}\left(x_{\lambda_{k}}^{i}\right)\right)$ is singular, then $x \neq 0$ exists such that

$$
\left(A-D\left(x_{\lambda_{k}}^{i}\right)+\lambda_{k} D^{\prime}\left(x_{\lambda_{k}}^{i}\right)\right) x=0 .
$$

Thus, we have the following contradiction

$$
\begin{aligned}
x^{T} x< & x^{T} A^{T} A x=(A x)^{T} A x \\
= & x^{T}\left(D\left(x_{\lambda_{k}}^{i}\right)-\lambda_{k} D^{\prime}\left(x_{\lambda_{k}}^{i}\right)\right)^{T} \\
& \times\left(D-\lambda_{k} D^{\prime}\left(x_{\lambda_{k}}^{i}\right)\right) x \leq x^{T} x
\end{aligned}
$$

for $\ell=1$ and $\lambda_{k} \in(1,2)$, where the first inequality follows from Lemma 5. Hence, $\left(A-D\left(x_{\lambda_{k}}^{i}\right)+\lambda_{k} D^{\prime}\left(x_{\lambda_{k}}^{i}\right)\right)$ is nonsingular.

We now establish boundness of the generalized Newton iteration (45) and thus existence of an accumulation.

Theorem 13. Let the singular values of $A \in \mathfrak{R}^{n \times n}$ exceed 1 . Then the iteration $x_{\lambda_{k}}^{i+1}=\left[A-D\left(x_{\lambda_{k}}^{i}\right)+\lambda_{k} D^{\prime}\left(x_{\lambda_{k}}^{i}\right)\right] \backslash(b+$ $\left.\lambda_{k}((1 / \ell)-1)\left[x_{\lambda_{k}}^{i}\right]_{+}^{1 / \ell}\right)$ of the Algorithm 11 is well defined and bounded. Consequently, there exists an accumulation point $\bar{x}_{k}$ such that $\left(A-D\left(\bar{x}_{k}\right)+\lambda_{k} D^{\prime}\left(\bar{x}_{k}\right)\right) \bar{x}_{k}=b+\lambda_{k}((1 / \ell)-1)\left[\bar{x}_{k}\right]_{+}^{1 / \ell}$.

Proof. By Lemma $12,\left(A-D\left(x_{\lambda_{k}}^{i}\right)+\lambda_{k} D^{\prime}\left(x_{\lambda_{k}}^{i}\right)\right)^{-1}$ exists; hence, the generalized Newton iteration $x_{\lambda_{k}}^{i+1}=\left[A-D\left(x_{\lambda_{k}}^{i}\right)+\right.$ $\left.\lambda_{k} D^{\prime}\left(x_{\lambda_{k}}^{i}\right)\right] \backslash\left(b+\lambda_{k}((1 / \ell)-1)\left[x_{\lambda}^{i}\right]_{+}^{1 / \ell}\right)$ is well defined.

We now prove the boundedness of the iterative sequence $\left\{x_{\lambda_{k}}^{i}\right\}$. Suppose that $\left\{x_{\lambda_{k}}^{i}\right\}$ is unbounded; then there exists a subsequence $\left\{x_{\lambda_{k}}^{i_{j}+1}\right\}$ with $\left\|x_{\lambda_{k}}^{i_{j}+1}\right\| \rightarrow \infty$ and nonzero such that $D\left(x_{\lambda_{k}}^{i_{j}}\right)=\widetilde{D}, D^{\prime}\left(x_{\lambda_{k}}^{i_{j}}\right)=\widetilde{D^{\prime}}$, where $\widetilde{D}$ and $\widetilde{D^{\prime}}$ are fixed diagonal matrices extended from the finite number of possible configurations for $D\left(x_{\lambda_{k}}^{i_{j}}\right)$ in the sequence $\left\{D\left(x_{\lambda_{k}}^{i_{j}}\right)\right\}$ and $D^{\prime}\left(x_{\lambda_{k}}^{i_{j}}\right)$ in the sequence $\left\{D^{\prime}\left(x_{\lambda_{k}}^{i_{j}}\right)\right\}$, respectively, such that the bounded subsequence $\left\{x_{\lambda_{k}}^{i_{j}+1} /\left\|x_{\lambda_{k}}^{i_{j}+1}\right\|\right\}$ converges to $\tilde{x}$. Hence

$$
\left(A-\widetilde{D}+\lambda_{k} \widetilde{D^{\prime}}\right) \frac{x_{\lambda_{k}}^{i_{j}+1}}{\left\|x_{\lambda_{k}}^{i_{j}+1}\right\|}=\frac{b+\lambda_{k}((1 / \ell)-1)\left[x_{\lambda}^{i}\right]_{+}^{1 / \ell}}{\left\|x_{\lambda_{k}}^{i_{j}+1}\right\|} .
$$

By letting $j \rightarrow \infty$, we obtain

$$
\left(A-\widetilde{D}+\lambda_{k} \widetilde{D^{\prime}}\right) \tilde{x}=0, \quad\|\widetilde{x}\|=1,
$$

since $\left\|x_{\lambda_{k}}^{i_{j}+1}\right\| \rightarrow \infty$. This contradicts the nonsingularity $\left(A-\widetilde{D}+\lambda_{k} \widetilde{D^{\prime}}\right)$ which follows from Lemma 12. Therefore, the iterative sequence $\left\{x_{\lambda_{k}}^{i}\right\}$ is bounded and there exists an accumulation point $\bar{x}_{k}$ such that $\left(A-D\left(\bar{x}_{k}\right)+\lambda_{k} D^{\prime}\left(\bar{x}_{k}\right)\right) \bar{x}_{k}=$ $b+\lambda_{k}((1 / \ell)-1)\left[\bar{x}_{k}\right]_{+}^{1 / \ell}$.

We then establish the finite termination of generalized Newton iteration (45).

Theorem 14. Let the singular values of $A \in \mathfrak{R}^{n \times n}$ exceed 1. If $D\left(x_{\lambda_{k}}^{i+1}\right)=D\left(x_{\lambda_{k}}^{i}\right), D^{\prime}\left(x_{\lambda_{k}}^{i+1}\right)=D^{\prime}\left(x_{\lambda_{k}}^{i}\right)$, and $\left\|x_{\lambda_{k}}^{i+1}-x_{\lambda_{k}}^{i}\right\| \leq \varepsilon$ for some $i$ for the well defined iteration (45) in Algorithm 11, then $x_{\lambda_{k}}^{i+1}$ solves the nonlinear penalized equation (10). 
Proof. The generalized Newton iteration

$$
\begin{aligned}
& x_{\lambda_{k}}^{i+1}=\left[A-D\left(x_{\lambda_{k}}^{i}\right)+\lambda_{k} D^{\prime}\left(x_{\lambda_{k}}^{i}\right)\right] \backslash\left(b+\lambda_{k}\left(\frac{1}{\ell}-1\right)\right. \\
&\left.\times\left[x_{\lambda_{k}}^{i}\right]_{+}^{1 / \ell}\right)
\end{aligned}
$$

is well defined by Lemma 12, and if $D\left(x_{\lambda_{k}}^{i+1}\right)=D\left(x_{\lambda_{k}}^{i}\right)$, $D^{\prime}\left(x_{\lambda_{k}}^{i+1}\right)=D^{\prime}\left(x_{\lambda_{k}}^{i}\right)$ and $\left\|x_{\lambda_{k}}^{i+1}-x_{\lambda_{k}}^{i}\right\| \leq \varepsilon$, then

$$
\begin{aligned}
0= & {\left[A-D\left(x_{\lambda_{k}}^{i}\right)+\lambda_{k} D^{\prime}\left(x_{\lambda_{k}}^{i}\right)\right] x_{\lambda_{k}}^{i+1} } \\
& -b-\lambda_{k}\left(\frac{1}{\ell}-1\right)\left[x_{\lambda_{k}}^{i}\right]_{+}^{1 / \ell} \\
= & A x_{\lambda_{k}}^{i+1}-D\left(x_{\lambda_{k}}^{i}\right) x_{\lambda_{k}}^{i+1}+\lambda_{k} D^{\prime}\left(x_{\lambda_{k}}^{i}\right) x_{\lambda_{k}}^{i+1} \\
& -b-\lambda_{k}\left(\frac{1}{\ell}-1\right)\left[x_{\lambda_{k}}^{i}\right]_{+}^{1 / \ell} \\
= & A x_{\lambda_{k}}^{i+1}-D\left(x_{\lambda_{k}}^{i+1}\right) x_{\lambda_{k}}^{i+1}+\lambda_{k} D^{\prime}\left(x_{\lambda_{k}}^{i+1}\right) x_{\lambda_{k}}^{i+1} \\
& -b-\lambda_{k}\left(\frac{1}{\ell}-1\right)\left[x_{\lambda_{k}}^{i}\right]_{+}^{1 / \ell} \\
= & A x_{\lambda_{k}}^{i+1}-\left|x_{\lambda_{k}}^{i+1}\right|+\lambda_{k}\left[x_{\lambda_{k}}^{i}\right]_{+}^{1 / \ell}-b \\
& +\lambda_{k}\left(D^{\prime}\left(x_{\lambda_{k}}^{i+1}\right) x_{\lambda_{k}}^{i+1}-D^{\prime}\left(x_{\lambda_{k}}^{i}\right) x_{\lambda_{k}}^{i}\right) \\
= & A x_{\lambda_{k}}^{i+1}-\left|x_{\lambda_{k}}^{i+1}\right|+\lambda_{k}\left[x_{\lambda_{k}}^{i}\right]_{+}^{1 / \ell}-b \\
& +\lambda_{k} D^{\prime}\left(x_{\lambda_{k}}^{i+1}\right)\left(x_{\lambda_{k}}^{i+1}-x_{\lambda_{k}}^{i}\right) \\
= & A x_{\lambda_{k}}^{i+1}-\left|x_{\lambda_{k}}^{i+1}\right|+\lambda_{k}\left[x_{\lambda_{k}}^{i+1}\right]_{+}^{1 / \ell}-b+O(\varepsilon),
\end{aligned}
$$

giving the result.

Furthermore, we have the following results.

Theorem 15. Suppose that the singular values of $A \in \mathfrak{R}^{n \times n}$ exceed 1 and $\bar{x}_{k}$ is the unique solution of the nonlinear penalized equation (10). Then, for any $x_{\lambda_{k}}$ such that

$$
\begin{aligned}
&\left(\bar{x}_{k}\right)_{i}>0 \Longrightarrow\left(x_{\lambda_{k}}\right)_{i}>0, \quad\left(\bar{x}_{k}\right)_{i}<0 \Longrightarrow\left(x_{\lambda_{k}}\right)_{i}<0, \\
& \forall i \in\{1,2, \ldots, n\},
\end{aligned}
$$

the generalized Newton iteration (45) reaches $\bar{x}_{k}$ in one iteration.

Proof. The theorem can be proved in a similar way to the one in [24, Lemma 2.1]. We omit it here.

Remark 16. Not that the "sign match" property (52) holds if $x_{\lambda_{k}}$ is sufficiently near $\bar{x}_{k}$. Hence, by Theorem 15, global convergence is then obvious for generalized Newton iteration (45).
Now, we discuss the globally linear convergence of $\left\{x_{\lambda_{k}}^{i}\right\}_{i=1}^{+\infty}$ generated by generalized Newton iteration (45).

Theorem 17. If $\left\|\left(A+\lambda_{k} D^{\prime}\left(x_{\lambda_{k}}^{i}\right)\right)^{-1}\right\|<1 /\left(4+\lambda_{k}(1+\right.$ $\left.\left.\left\|D^{\prime}\left(x_{\lambda_{k}}^{i}\right)\right\|\right)\right)$ holds for all sufficiently large $\lambda_{k}$ and $\left\|D\left(x_{\lambda_{k}}^{i}\right)\right\| \neq 0$, then the generalized Newton iteration (45) converges linearly from any starting point $x_{\lambda_{k}}^{0}$ to a solution $\bar{x}_{k}$ of the nonlinear penalized equation (10).

Proof. Since $\left\|\left(A+\lambda_{k} D^{\prime}\left(x_{\lambda_{k}}^{i}\right)\right)^{-1}\right\| \cdot\left\|D\left(x_{\lambda_{k}}^{i}\right)\right\|<1$ according to the assumption and the definition of $D\left(x_{\lambda_{k}}^{i}\right)$, then $(A+$ $\left.\lambda_{k} D^{\prime}\left(x_{\lambda_{k}}^{i}\right)-D\left(x_{\lambda_{k}}^{i}\right)\right)^{-1}$ exists for any $x_{\lambda_{k}}^{i}$ by Lemma 6 . We also have by the same lemma that

$$
\begin{aligned}
& \left\|\left[\left(A+\lambda_{k} D^{\prime}\left(x_{\lambda_{k}}^{i}\right)\right)-D\left(x_{\lambda_{k}}^{i}\right)\right]^{-1}\right\| \\
& \quad \leq \frac{\left\|\left(A+\lambda_{k} D^{\prime}\left(x_{\lambda_{k}}^{i}\right)\right)^{-1}\right\|}{1-\left\|\left(A+\lambda_{k} D^{\prime}\left(x_{\lambda_{k}}^{i}\right)\right)^{-1}\right\| \cdot\left\|D\left(x_{\lambda_{k}}^{i}\right)\right\|} \\
& \quad<\frac{1 /\left(4+\lambda_{k}\left(1+\left\|D^{\prime}\left(x_{\lambda_{k}}^{i}\right)\right\|\right)\right)}{1-\left(1 / 1 /\left(4+\lambda_{k}\left(1+\left\|D^{\prime}\left(x_{\lambda_{k}}^{i}\right)\right\|\right)\right)\right) \cdot 1} \\
& =\frac{1}{3+\lambda_{k}\left(1+\left\|D^{\prime}\left(x_{\lambda_{k}}^{i}\right)\right\|\right)} .
\end{aligned}
$$

Let $\bar{x}_{k}$ be a solution of the nonlinear penalized equation (10). To simplify notation, let $\bar{D}=D\left(\bar{x}_{k}\right), D^{i}=D\left(x_{\lambda_{k}}^{i}\right), \overline{D^{\prime}}=$ $D^{\prime}\left(\bar{x}_{k}\right)$, and ${D^{\prime}}^{i}=D^{\prime}\left(x_{\lambda_{k}}^{i}\right)$. Noting that $\left|\bar{x}_{k}\right|=\bar{D} \bar{x}_{k},\left|x_{\lambda_{k}}^{i}\right|=$ $D^{i} x_{\lambda_{k}}^{i},(1 / \ell)\left[\bar{x}_{k}\right]_{+}^{1 / \ell}=\overline{D^{\prime}} \bar{x}_{k}$, and $(1 / \ell)\left[x_{\lambda_{k}}^{i}\right]_{+}^{1 / \ell}=D^{\prime i} x_{\lambda_{k}}^{i}$, we have

$$
\begin{aligned}
A\left(x_{\lambda_{k}}^{i+1}-\bar{x}_{k}\right) & D^{i} x_{\lambda_{k}}^{i+1}-\lambda_{k} D^{\prime i} x_{\lambda_{k}}^{i+1}+b+\lambda_{k}\left(\frac{1}{\ell}-1\right)\left[x_{\lambda_{k}}^{i}\right]_{+}^{1 / \ell} \\
& -\left(\bar{D} \bar{x}_{k}-\lambda_{k} \overline{D^{\prime}} \bar{x}_{k}+b+\lambda_{k}\left(\frac{1}{\ell}-1\right)\left[\bar{x}_{k}\right]_{+}^{1 / \ell}\right) \\
= & D^{i} x_{\lambda_{k}}^{i+1}-\lambda_{k} D^{\prime i} x_{\lambda_{k}}^{i+1}-\bar{D} \bar{x}_{k}+\lambda_{k} \overline{D^{\prime}} \bar{x}_{k} \\
& +\lambda_{k}\left(\frac{1}{\ell}-1\right)\left(\left[x_{\lambda_{k}}^{i}\right]_{+}^{1 / \ell}-\left[\bar{x}_{k}\right]_{+}^{1 / \ell}\right) \\
= & \left(D^{i} x_{\lambda_{k}}^{i+1}-\bar{D} \bar{x}_{k}\right)-\lambda_{k}\left(D^{\prime i} x_{\lambda_{k}}^{i+1}-\overline{D^{\prime}} \bar{x}_{k}\right) \\
& +\lambda_{k}\left(\frac{1}{\ell}-1\right)\left(\left[x_{\lambda_{k}}^{i}\right]_{+}^{1 / \ell}-\left[\bar{x}_{k}\right]_{+}^{1 / \ell}\right) \\
= & \left(D^{i} x_{\lambda_{k}}^{i+1}-\left|\bar{x}_{k}\right|\right)-\lambda_{k}\left(D^{\prime i} x_{\lambda_{k}}^{i+1}-\frac{1}{\ell}\left[\bar{x}_{k}\right]_{+}^{1 / \ell}\right) \\
& +\lambda_{k}\left(\frac{1}{\ell}-1\right)\left(\left[x_{\lambda_{k}}^{i}\right]_{+}^{1 / \ell}-\left[\bar{x}_{k}\right]_{+}^{1 / \ell}\right)
\end{aligned}
$$




$$
\begin{aligned}
= & D^{i}\left(x_{\lambda_{k}}^{i+1}-x_{\lambda_{k}}^{i}+x_{\lambda_{k}}^{i}\right)-\left|\bar{x}_{k}\right| \\
& -\lambda_{k}\left[D^{\prime i}\left(x_{\lambda_{k}}^{i+1}-x_{\lambda_{k}}^{i}+x_{\lambda_{k}}^{i}\right)-\frac{1}{\ell}\left[\bar{x}_{k}\right]_{+}^{1 / \ell}\right] \\
& +\lambda_{k}\left(\frac{1}{\ell}-1\right)\left(\left[x_{\lambda_{k}}^{i}\right]_{+}^{1 / \ell}-\left[\bar{x}_{k}\right]_{+}^{1 / \ell}\right) \\
= & \left|x_{\lambda_{k}}^{i}\right|-\left|\bar{x}_{k}\right|+D^{i}\left(x_{\lambda_{k}}^{i+1}-\bar{x}_{k}+\bar{x}_{k}-x_{\lambda_{k}}^{i}\right) \\
& -\frac{1}{\ell} \lambda_{k}\left(\left[x_{\lambda_{k}}^{i}\right]_{+}^{1 / \ell}-\left[\bar{x}_{k}\right]_{+}^{1 / \ell}\right) \\
& -\lambda_{k} D^{\prime i}\left(x_{\lambda_{k}}^{i+1}-\bar{x}_{k}+\bar{x}_{k}-x_{\lambda_{k}}^{i}\right) \\
& +\lambda_{k}\left(\frac{1}{\ell}-1\right)\left(\left[x_{\lambda_{k}}^{i}\right]_{+}^{1 / \ell}-\left[\bar{x}_{k}\right]_{+}^{1 / \ell}\right)
\end{aligned}
$$

Applying Lemmas 7 and 8 and $\left\|\left[x_{\lambda_{k}}^{i}\right]_{+}-\left[\bar{x}_{k}\right]_{+}\right\| \leq \varepsilon$ when $i$ is sufficiently large, we get

$$
\begin{aligned}
& \left\|x_{\lambda_{k}}^{i+1}-\bar{x}_{k}\right\| \\
& \leq\left\|\left(A-D^{i}+\lambda_{k} D^{\prime i}\right)^{-1}\right\| \\
& \cdot\left(\left(3+\frac{\lambda_{k}}{\ell}\right)\left\|x_{\lambda_{k}}^{i}-\bar{x}_{k}\right\|\right. \\
& +\lambda_{k}\left\|D^{\prime i}\left(x_{\lambda_{k}}^{i}-\bar{x}_{k}\right)\right\| \\
& \left.+\lambda_{k}\left(1-\frac{1}{\ell}\right)\left\|\left[x_{\lambda_{k}}^{i}\right]_{+}-\left[\bar{x}_{k}\right]_{+}\right\|+O(\varepsilon)\right) \\
& \leq\left(3+\lambda_{k}+\lambda_{k}\left\|D^{\prime i}\right\|+O(\varepsilon)\right) \\
& \cdot\left\|\left(A-D^{i}+\lambda_{k} D^{\prime i}\right)^{-1}\right\| \cdot\left\|x_{\lambda_{k}}^{i}-\bar{x}_{k}\right\| .
\end{aligned}
$$

where the first inequality follows from $\left(A-\bar{D}+\lambda_{k} \overline{D^{\prime}}\right) \bar{x}_{k}=$ $b+\lambda_{k}((1 / \ell)-1)\left[\bar{x}_{k}\right]_{+}^{1 / \ell}$ and $\left(A-D^{i}+\lambda_{k} D^{\prime i}\right) x_{\lambda_{k}}^{i+1}=b+\lambda_{k}((1 / \ell)-$ 1) $\left[x_{\lambda_{k}}^{i}\right]_{+}^{1 / \ell}$.

Hence, one has that

$$
\begin{aligned}
(A & \left.-D^{i}+\lambda_{k} D^{\prime i}\right)\left(x_{\lambda_{k}}^{i+1}-\bar{x}_{k}\right) \\
= & \left|x_{\lambda_{k}}^{i}\right|-\left|\bar{x}_{k}\right|-D^{i}\left(x_{\lambda_{k}}^{i}-\bar{x}_{k}\right) \\
& -\frac{1}{\ell} \lambda_{k}\left(\left[x_{\lambda_{k}}^{i}\right]_{+}-\left[\bar{x}_{k}\right]_{+}\right)+\lambda_{k} D^{\prime i}\left(x_{\lambda_{k}}^{i}-\bar{x}_{k}\right) \\
& +\lambda_{k}\left(\frac{1}{\ell}-1\right)\left(\left[x_{\lambda_{k}}^{i}\right]_{+}^{1 / \ell}-\left[\bar{x}_{k}\right]_{+}^{1 / \ell}\right) .
\end{aligned}
$$

Letting $i \rightarrow \infty, \varepsilon \rightarrow 0$ and taking limits in both sides of the last inequality above, we have

$$
\begin{aligned}
\frac{\left\|x_{\lambda_{k}}^{i+1}-\bar{x}_{k}\right\|}{\left\|x_{\lambda_{k}}^{i}-\bar{x}_{k}\right\|} \leq & \left(3+\lambda_{k}\left(1+\left\|D^{\prime}\left(x_{\lambda_{k}}^{i}\right)\right\|\right)\right) \\
& \cdot\left\|\left(A-D^{i}\left(x_{\lambda_{k}}^{i}\right)+\lambda_{k} D^{\prime i}\left(x_{\lambda_{k}}^{i}\right)\right)^{-1}\right\|<1,
\end{aligned}
$$

Thus

$$
\begin{aligned}
x_{\lambda_{k}}^{i+1}- & \bar{x}_{k} \\
=(A & \left.-D^{i}+\lambda_{k} D^{\prime i}\right)^{-1} \\
& \times\left[\left|x_{\lambda_{k}}^{i}\right|-\left|\bar{x}_{k}\right|-D^{i}\left(x_{\lambda_{k}}^{i}-\bar{x}_{k}\right)\right. \\
& \quad-\frac{1}{\ell} \lambda_{k}\left(\left[x_{\lambda_{k}}^{i}\right]_{+}-\left[\bar{x}_{k}\right]_{+}\right) \\
& +\lambda_{k} D^{\prime i}\left(x_{\lambda_{k}}^{i}-\bar{x}_{k}\right) \\
& \left.+\lambda_{k}\left(\frac{1}{\ell}-1\right)\left(\left[x_{\lambda_{k}}^{i}\right]_{+}^{1 / \ell}-\left[\bar{x}_{k}\right]_{+}^{1 / \ell}\right)\right] .
\end{aligned}
$$

In the above proof, the choice of $\bar{x}_{k}$ is arbitrary; hence we have the following result.

Corollary 18. Assume that $\left\|\left(A+\lambda_{k} D^{\prime}\left(x_{\lambda_{k}}^{i}\right)\right)^{-1}\right\|<1 /(1+$ $\left.\lambda_{k}^{i}\left(1+\left\|D^{\prime}\left(x_{\lambda_{k}}^{i}\right)\right\|\right)\right)$ and $\left\|D\left(x_{\lambda_{k}}^{i}\right)\right\| \neq 0$ for any sufficiently large $\lambda_{k}$. Then the nonlinear penalized equation (10) has a unique solution for any $b \in \mathfrak{R}^{n}$.

Finally, we give the globally superlinear convergence of $\left\{x_{\lambda_{k}}^{i}\right\}_{i=1}^{+\infty}$ generated by generalized Newton iteration (45).

Theorem 19. Assume that $\left\|\left(A+\lambda_{k} D^{\prime}\left(x_{\lambda_{k}}^{i}\right)\right)^{-1}\right\|<1 /(1+$ $\left.\lambda_{k}^{i}\left(1+\left\|D^{\prime}\left(x_{\lambda_{k}}^{i}\right)\right\|\right)\right)$ and $\left\|D\left(x_{\lambda_{k}}^{i}\right)\right\| \neq 0$ for any sufficiently large $\lambda_{k}$. Then the generalized Newton iteration (45) converges superlinearly from any starting point $x_{\lambda_{k}}^{0}$ to a solution $\bar{x}_{k}$ of the nonlinear penalized equation (10). 
Proof. According to Lemma 6, one has that

$$
\begin{aligned}
& \left\|\left[\left(A+\lambda_{k} D^{\prime}\left(x_{\lambda_{k}}^{i}\right)\right)-D\left(x_{\lambda_{k}}^{i}\right)\right]^{-1}\right\| \\
& \leq \frac{\left\|\left(A+\lambda_{k} D^{\prime}\left(x_{\lambda_{k}}^{i}\right)\right)^{-1}\right\|}{1-\left\|\left(A+\lambda_{k} D^{\prime}\left(x_{\lambda_{k}}^{i}\right)\right)^{-1}\right\| \cdot\left\|D\left(x_{\lambda_{k}}^{i}\right)\right\|} \\
& \leq \frac{\left\|\left(A+\lambda_{k} D^{\prime}\left(x_{\lambda_{k}}^{i}\right)\right)^{-1}\right\|}{1-\left\|\left(A+\lambda_{k} D^{\prime}\left(x_{\lambda_{k}}^{i}\right)\right)^{-1}\right\|} \\
& <\frac{1}{\lambda_{k}^{i}\left(1+\left\|D^{\prime}\left(x_{\lambda_{k}}^{i}\right)\right\|\right)},
\end{aligned}
$$

where the last inequality follows from the sequence $\|(A+$ $\left.\lambda_{k} D^{\prime}\left(x_{\lambda_{k}}^{i}\right)\right)^{-1} \| \leq 1 /\left(1+\lambda_{k}^{i}\left(1+\left\|D^{\prime}\left(x_{\lambda_{k}}^{i}\right)\right\|\right)\right)$.

Combining this with (58) in Theorem 15, we have

$$
\begin{aligned}
\frac{\left\|x_{\lambda_{k}}^{i+1}-\bar{x}_{k}\right\|}{\left\|x_{\lambda_{k}}^{i}-\bar{x}_{k}\right\|}< & \left(3+\lambda_{k}\left(1+\left\|D^{\prime}\left(x_{\lambda_{k}}^{i}\right)\right\|\right)\right) \\
& \cdot\left\|\left(A-D\left(x_{\lambda_{k}}^{i}\right)+\lambda_{k} D^{\prime}\left(x_{\lambda_{k}}^{i}\right)\right)^{-1}\right\| \\
< & \frac{3+\lambda_{k}\left(1+\left\|D^{\prime}\left(x_{\lambda_{k}}^{i}\right)\right\|\right)}{\lambda_{k}^{i}\left(1+\left\|D^{\prime}\left(x_{\lambda_{k}}^{i}\right)\right\|\right)} .
\end{aligned}
$$

Letting $i \rightarrow \infty$ and taking limits in (61), we can see that the sequence $\left\{x_{\lambda_{k}}^{i}\right\}_{i=1}^{+\infty}$ generated by generalized Newton iteration (45) converges superlinearly to a solution $\bar{x}_{k}$.

4.2. Convergence of Penalized-Equation-Based Generalized Newton Method. In this subsection, we will focus on the convergence of Algorithm 11. We first present the global convergence.

Theorem 20. Let the singular values of $A \in \mathfrak{R}^{n \times n}$ exceed 1 . Then the sequence $\left\{\bar{x}_{k}\right\}$ generated by Algorithm 11 is bounded. Consequently, there exists an accumulation point $\bar{x}^{*}$ of the nonlinear penalized equation (10).

Proof. Since $\bar{x}_{k}$ is an accumulation point of $\left\{x_{\lambda_{k}}^{i}\right\}_{i=1}^{+\infty}$, it follows from Theorem 13 that the sequence $\left\{x_{\lambda_{k}}^{i}\right\}_{i=1}^{+\infty}$ is bounded, we can thus obtain the boundness of $\left\{\bar{x}_{k}\right\}$. Hence there exists an accumulation point $\bar{x}^{*}$ of $\left\{\bar{x}_{k}\right\}$ such that the nonlinear penalized equation (10) holds, giving the results.

We then establish the linear convergence of Algorithm 11.

Theorem 21. Under the assumption that $\left\|\left(A+\lambda_{k} D^{\prime}\left(\bar{x}_{k}\right)\right)^{-1}\right\|<$ $1 /\left(1+\lambda_{k}\left(1+\left\|D^{\prime}\left(\bar{x}_{k}\right)\right\|\right)\right),\left\|D\left(\bar{x}_{k}\right)\right\| \neq 0$, Algorithm 11 converges linearly from any starting point $x_{\lambda_{0}}^{0}$ to a solution $\bar{x}^{*}$ of the nonlinear penalized equation (10).

Proof. Taking into account "match property" (52), the theorem can be proved in a similar way to that of Theorem 15 .
TABLE 1: The numerical results of Example 1.

\begin{tabular}{lcc}
\hline Initial point $x_{\lambda_{0}}^{s}$ & Numbers of Newton iterations & $\bar{x}^{*}$ \\
\hline$(0,0)^{T}$ & 2 & $\left(-\frac{4}{11},-\frac{1}{11}\right)^{T}$ \\
\hline$(0,1)^{T}$ & 2 & $\left(-\frac{4}{11},-\frac{1}{11}\right)^{T}$ \\
\hline$(-1,-1)^{T}$ & 1 & $\left(-\frac{4}{11},-\frac{1}{11}\right)^{T}$ \\
\hline$(2,-1)^{T}$ & 2 & $\left(-\frac{4}{11},-\frac{1}{11}\right)^{T}$ \\
\hline
\end{tabular}

Finally, we establish the superlinear convergence of Algorithm 11.

Theorem 22. Under the assumption that $\|(A+$ $\left.\lambda_{k} D^{\prime}\left(\bar{x}_{k}\right)\right)^{-1} \|<1 /\left(1+\lambda_{k}^{m}\left(1+\left\|D^{\prime}\left(\bar{x}_{k}\right)\right\|\right)\right)(m>1)$, $\left\|D\left(\bar{x}_{k}\right)\right\| \neq 0$, Algorithm 11 converges superlinearly from any starting point $x_{\lambda_{0}}^{0}$ to a solution $\bar{x}^{*}$ of the nonlinear penalized equation (10).

Proof. Taking into account "match property" (52), the theorem can be proved in a similar way to that of Theorems 15 and 17.

\section{Numerical Results}

In this section, we consider several examples to show the efficiency of the proposed method by running in MATLAB 7.5 with Intel(R) Core (TM) of $2 \times 2.70 \mathrm{GHz}$ and RAM of 2.0 GB. Throughout these computational experiments, the parameters used in the algorithm are set as $\varepsilon=10^{-6}, \lambda_{0}=10$, $\mu=2$, and $\ell=1$. The accumulation point of Algorithm 11 is written as $\bar{x}^{*}$.

Example 1. Let the matrix $A$ of $\operatorname{AVLCP}(A, b)$ be given by

$$
A=\left(\begin{array}{cc}
2 & -1 \\
-1 & 3
\end{array}\right)
$$

and $b=(-1,0)^{T}$. The solution of $\operatorname{AVLCP}(A, b)$ is $x^{*}=$ $(-4 / 11,-1 / 11)^{T}$. The computational results are shown in Table 1 .

Example 2. Let the matrix $A$ of $\operatorname{AVLCP}(A, b)$ be given by

$$
A=\left(\begin{array}{cccc}
4 & -1 & 0 & 0 \\
-1 & 4 & -1 & 0 \\
0 & -1 & 4 & -1 \\
0 & 0 & -1 & 4
\end{array}\right)
$$

and $b=(-1,-1,0,0)^{T}$. The solution of $\operatorname{AVLCP}(A, b)$ is $x^{*}=(-139 / 551,-144 / 551,-30 / 551,-6 / 551)^{T}$. The computational results are shown in Table 2. 
TABLE 2: The numerical results of Example 2.

\begin{tabular}{lcc}
\hline Initial point $x_{\lambda_{0}}^{s}$ & $\begin{array}{c}\text { Numbers } \\
\text { of } \\
\text { Newton } \\
\text { iterations }\end{array}$ & $\bar{x}^{*}$ \\
\hline$(-1,-1,-1,-1)^{T}$ & 1 & $\left(-\frac{139}{551},-\frac{144}{551},-\frac{30}{551},-\frac{6}{551}\right)^{T}$ \\
\hline$(0,0,0,0)^{T}$ & 2 & $\left(-\frac{139}{551},-\frac{144}{551},-\frac{30}{551},-\frac{6}{551}\right)^{T}$ \\
\hline$(1,-1,1,-1)^{T}$ & 2 & $\left(-\frac{139}{551},-\frac{144}{551},-\frac{30}{551},-\frac{6}{551}\right)^{T}$ \\
\hline$(1,2,-1,0)^{T}$ & 2 & $\left(-\frac{139}{551},-\frac{144}{551},-\frac{30}{551},-\frac{6}{551}\right)^{T}$ \\
\hline
\end{tabular}

Example 3. Let the matrix $A$ of $\operatorname{AVLCP}(A, b)$ be given by

$$
A=\left(\begin{array}{cccccc}
4 & 0 & 0 & 0 & -1 & -1 \\
0 & 4 & 0 & 0 & -1 & -1 \\
0 & 0 & 4 & -1 & -1 & 0 \\
0 & 0 & -1 & 4 & 0 & 0 \\
-1 & -1 & -1 & 0 & 4 & 0 \\
-1 & -1 & 0 & 0 & 0 & 4
\end{array}\right)
$$

and $b=(-4,4,-4,-4,4,-4)^{T}$. The solution of $\operatorname{AVLCP}(A, b)$ is $x^{*}=(-1,0,-1,-1,0,-1)^{T}$. The computational results are shown in Table 3.

Example 4. Let the matrix $A$ of $\operatorname{AVLCP}(A, b)$ be given by

$$
a_{i j}= \begin{cases}8, & \text { for } j=i, \\
-1, & \text { for }\left\{\begin{aligned}
j=i+1, i=1,2, \ldots, n-1, \\
j=i-1, i=2,3, \ldots, n,
\end{aligned}\right. \\
0, & \text { otherwise. }\end{cases}
$$

Let $b=(6,5,5, \ldots, 5,6)^{T}$. Then choose initial point $x_{\lambda_{0}}^{s}$ as $x_{\lambda_{0}}^{s}=(0,0, \ldots, 0)^{T}$. We compared our algorithm with the existing methods in $[15,19]$. The computational results are shown in Table 4.

From Table 1 to Table 4, we can see that our method has some nice convergence which coincides with our results.

\section{Conclusion}

In this paper, we propose a new approximation to absolutevalue linear complementarity problems (3) by using the nonlinear penalized equation (10), based on which a generalized Newton method is proposed for solving this penalized equation. Under suitable assumptions, the algorithm is shown to be both globally and superlinearly convergent. The numerical results presented showed that the generalized Newton method proposed by us is efficient. The results and ideas of this paper may be used to solve the

\begin{tabular}{|c|c|c|}
\hline Initial point $x_{\lambda_{0}}^{s}$ & $\begin{array}{c}\text { Numbers } \\
\text { of } \\
\text { Newton } \\
\text { iterations }\end{array}$ & $\bar{x}^{*}=\left(\bar{u}_{1}^{*}, \bar{u}_{2}^{*}, \bar{u}_{3}^{*}, \bar{u}_{4}^{*}, \bar{u}_{5}^{*}, \bar{u}_{6}^{*}\right)^{T}$ \\
\hline \multirow{6}{*}{$(0,0,0,0,0,0)^{T}$} & \multirow{6}{*}{21} & $\bar{u}_{1}^{*}=-1$ \\
\hline & & $\bar{u}_{2}^{*}=5.72204 e-7$ \\
\hline & & $\bar{u}_{3}^{*}=-1$ \\
\hline & & $\bar{u}_{4}^{*}=-1$ \\
\hline & & $\bar{u}_{5}^{*}=3.8147 e-7$ \\
\hline & & $\bar{u}_{6}^{*}=-1$ \\
\hline \multirow{6}{*}{$(-1,-1,-1,-1,-1,-1)^{T}$} & \multirow{6}{*}{21} & $\bar{u}_{1}^{*}=-1$ \\
\hline & & $\bar{u}_{2}^{*}=5.72204 e-7$ \\
\hline & & $\bar{u}_{3}^{*}=-1$ \\
\hline & & $\bar{u}_{4}^{*}=-1$ \\
\hline & & $\bar{u}_{5}^{*}=3.8147 e-7$ \\
\hline & & $\bar{u}_{6}^{*}=-1$ \\
\hline \multirow{6}{*}{$(-2,0,-2,-2,0,-2)^{T}$} & \multirow{6}{*}{20} & $\bar{u}_{1}^{*}=-1$ \\
\hline & & $\bar{u}_{2}^{*}=5.72204 e-7$ \\
\hline & & $\bar{u}_{3}^{*}=-1$ \\
\hline & & $\bar{u}_{4}^{*}=-1$ \\
\hline & & $\bar{u}_{5}^{*}=3.8147 e-7$ \\
\hline & & $\bar{u}_{6}^{*}=-1$ \\
\hline \multirow{6}{*}{$(1,1,1,1,1,1)^{T}$} & \multirow{6}{*}{21} & $\bar{u}_{1}^{*}=-1$ \\
\hline & & $\bar{u}_{2}^{*}=5.72204 e-7$ \\
\hline & & $\bar{u}_{3}^{*}=-1$ \\
\hline & & $\bar{u}_{4}^{*}=-1$ \\
\hline & & $\bar{u}_{5}^{*}=3.8147 e-7$ \\
\hline & & $\bar{u}_{6}^{*}=-1$ \\
\hline
\end{tabular}

TABLE 3: The numerical results of Example 3.

TABLE 4: Computational results of Example 4.

\begin{tabular}{lccc}
\hline \multirow{2}{*}{ Order } & \multicolumn{3}{c}{ Numbers of iterations } \\
& Iterative method [19] & GAOR method [15] & Algorithm 11 \\
\hline 4 & 10 & 10 & 8 \\
8 & 11 & 11 & 9 \\
16 & 11 & 11 & 9 \\
32 & 12 & 11 & 9 \\
64 & 12 & 11 & 9 \\
128 & 12 & 11 & 9 \\
256 & 12 & 11 & 9 \\
512 & 12 & 11 & 9 \\
1024 & 13 & 11 & 9 \\
\hline
\end{tabular}

absolute variational inequalities and related optimization problems.

\section{Conflict of Interests}

The authors declare that there is no conflict of interests regarding the publication of this paper. 


\section{References}

[1] R. W. Cottle, J.-S. Pang, and R. E. Stone, The Linear Complementarity Problems, Academic Press, San Diego, Calif, USA, 1992.

[2] F. Facchinei and J.-S. Pang, Finite-Dimensional Variational Inequalities and Complementarity Problems, Springer, New York, NY, USA, 2003.

[3] R. Glowinski, Numerical Methods for Nonlinear Variational Problems, Springer, New York, NY, USA, 1984.

[4] J. Nocedal and S. Wright, Numerical Optimization, Springer, New York, NY, USA, 1999.

[5] L. Qi and J. Sun, "A nonsmooth version of Newton's method," Mathematical Programming, vol. 58, no. 1-3, pp. 353-367, 1993.

[6] L. Q. Qi, "Convergence analysis of some algorithms for solving nonsmooth equations," Mathematics of Operations Research, vol. 18, no. 1, pp. 227-244, 1993.

[7] A. Fischer, "A special Newton-type optimization method," Optimization, vol. 24, no. 3-4, pp. 269-284, 1992.

[8] F. Facchinei and C. Kanzow, "A nonsmooth inexact Newton method for the solution of large-scale nonlinear complementarity problems," Mathematical Programming, vol. 76, no. 3, pp. 493-512, 1997.

[9] T. de Luca, F. Facchinei, and C. Kanzow, "A semismooth equation approach to the solution of nonlinear complementarity problems," Mathematical Programming, vol. 75, pp. 407-439, 1996.

[10] N. Yamashita and M. Fukushima, "Modified Newton methods for solving a semismooth reformulation of monotone complementarity problems," Mathematical Programming, vol. 76, pp. 469-491, 1997.

[11] B. Chen, X. Chen, and C. Kanzow, "A penalized FischerBurmeister NCP-function," Mathematical Programming, vol. 88, pp. 211-216, 2000.

[12] C. Kanzow and H. Kleinmichel, "A new class of semismooth Newton-type methods for nonlinear complementarity problems," Computational Optimization and Applications, vol. 11, no. 3, pp. 227-251, 1998.

[13] C. Kanzow, "Inexact semismooth Newton methods for largescale complementarity problems," Optimization Methods \& Software, vol. 19, no. 3-4, pp. 309-325, 2004.

[14] K. Ito and K. Kunisch, "On a semi-smooth Newton method and its globalization," Mathematical Programming, vol. 118, no. 2, pp. 347-370, 2009.

[15] M. A. Noor, J. Iqbal, K. I. Noor, and E. Al-Said, "Generalized AOR method for solving absolute complementarity problems," Journal of Applied Mathematics, vol. 2012, Article ID 743861, 14 pages, 2012.

[16] S. Wang, X. Q. Yang, and K. L. Teo, "Power penalty method for a linear complementarity problem arising from American option valuation," Journal of Optimization Theory and Applications, vol. 129, no. 2, pp. 227-254, 2006.

[17] S. Wang and X. Q. Yang, "A power penalty method for linear complementarity problems," Operations Research Letters, vol. 36, no. 2, pp. 211-214, 2008.

[18] S. Wang and C. S. Huang, "A power penalty method for solving a nonlinear parabolic complementarity problem," Nonlinear Analysis, vol. 69, no. 4, pp. 1125-1137, 2008.

[19] M. A. Noor, J. Iqbal, K. I. Noor, and E. Al-Said, "On an iterative method for solving absolute value equations," Optimization Letters, vol. 6, no. 5, pp. 1027-1033, 2012.
[20] R. T. Rockafellar, Convex Analysis, Princeton University Press, Princeton, NJ, USA, 1970.

[21] J. M. Ortega and W. C. Rheinboldt, Iterative Solution of Nonlinear Equations in Several Variables, Academic Press, New York, NY, USA, 1970.

[22] O. L. Mangasarian, "Absolute value programming," Computational Optimization and Applications, vol. 36, no. 1, pp. 43-53, 2007.

[23] O. L. Mangasarian, "A generalized Newton method for absolute value equations," Optimization Letters, vol. 3, no. 1, pp. 101-108, 2009.

[24] C. Zhang and Q. J. Wei, "Global and finite convergence of a generalized Newton method for absolute value equations," Journal of Optimization Theory and Applications, vol. 143, no. 2, pp. 391-403, 2009. 


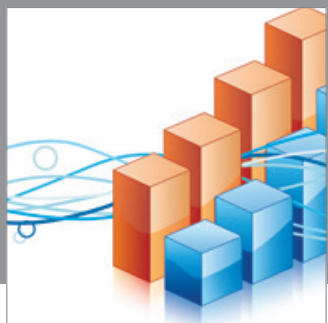

Advances in

Operations Research

mansans

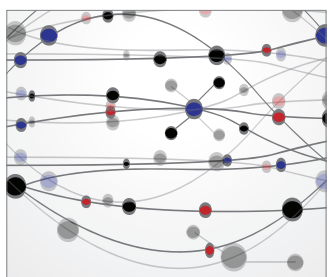

The Scientific World Journal
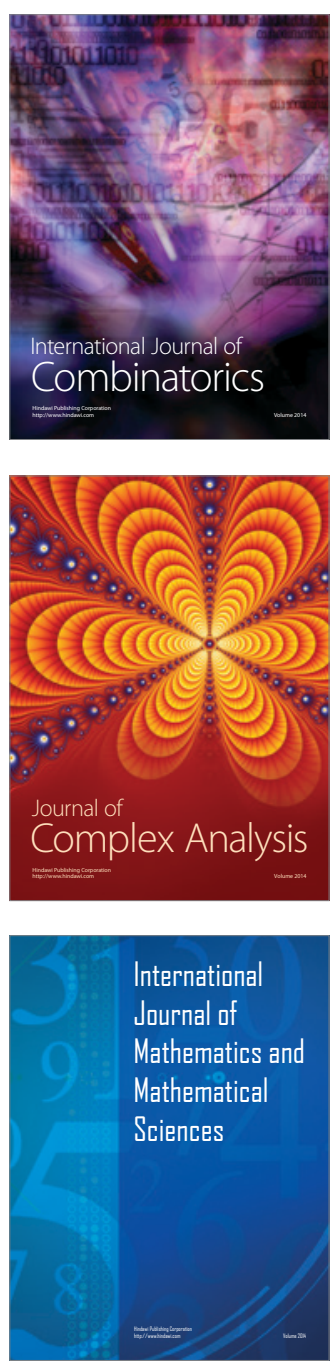
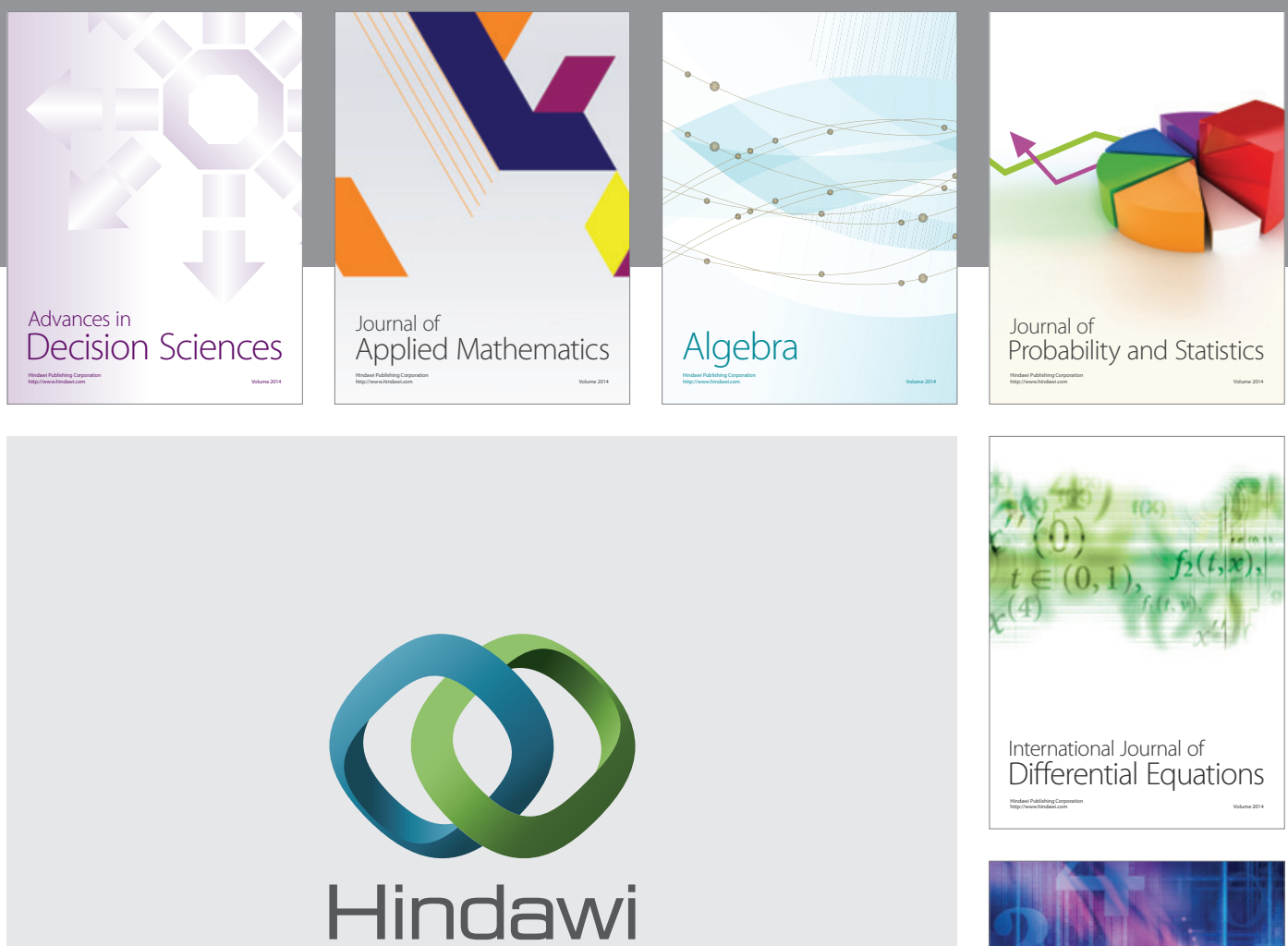

Submit your manuscripts at http://www.hindawi.com
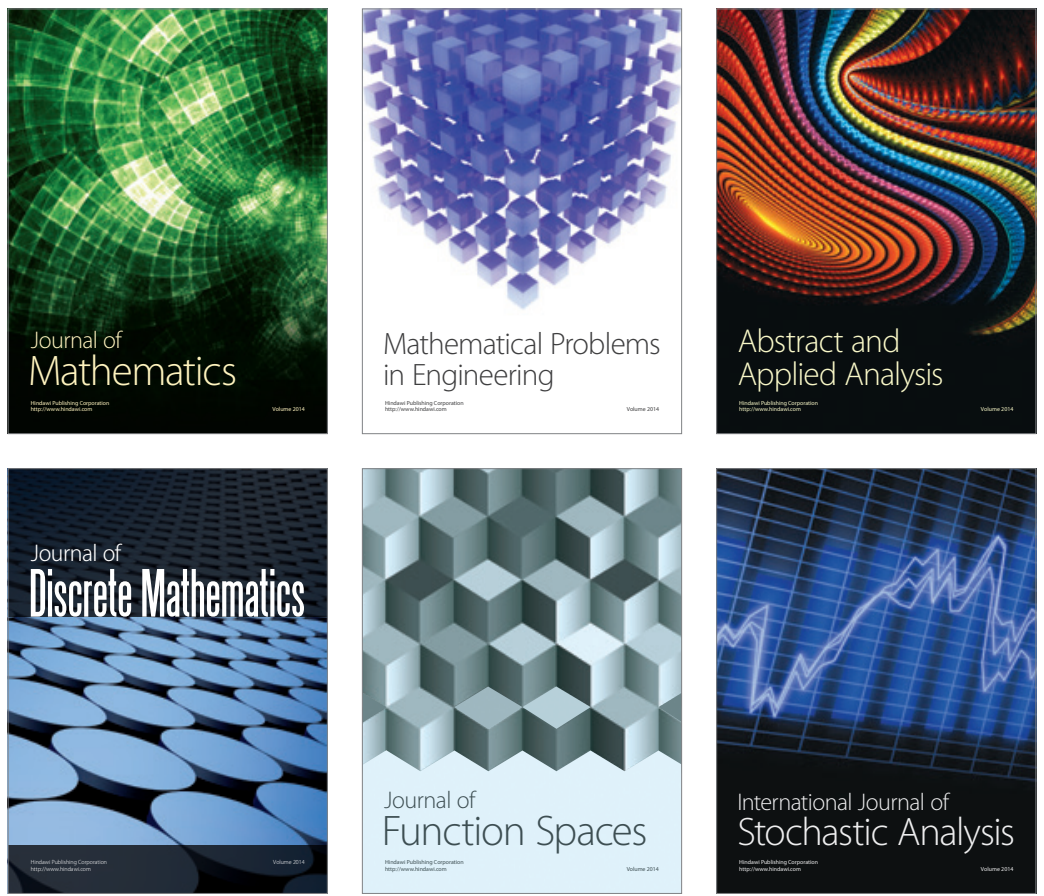

Journal of

Function Spaces

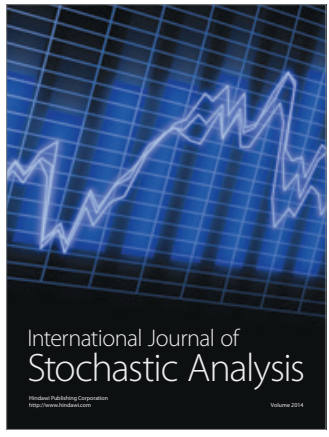

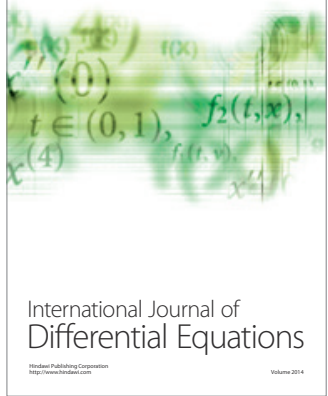
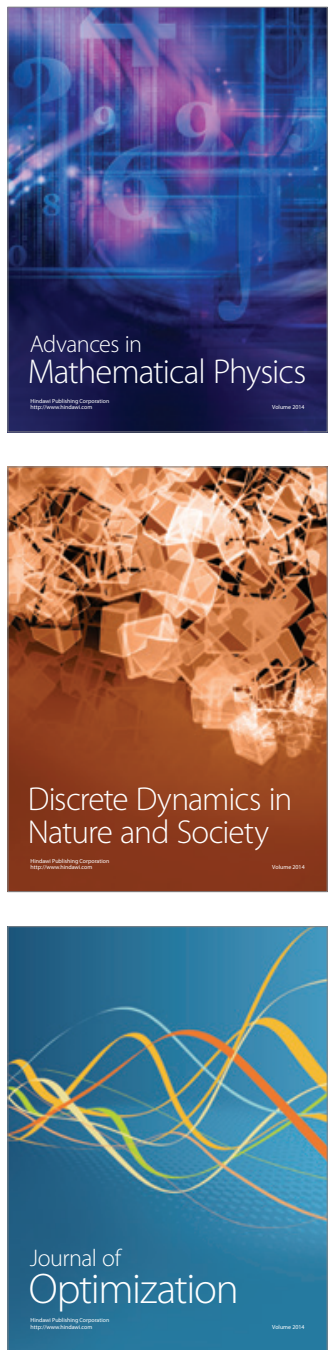